\title{
Appendicular desmoid tumour, an uncommon cause for abdominal pain
}

\author{
Vladimir Revicky • Mazen Freij • Jose Nieto • \\ Edward P. Morris
}

Received: 23 December 2008 / Accepted: 20 January 2009/Published online: 7 February 2009

(C) Springer-Verlag 2009

\begin{abstract}
This case report refers to a 17 -year-old woman who was admitted to a gynaecological ward with severe lower abdominal pain. She underwent an explorative laparotomy, which revealed a large mass arising from the appendix. Her uterus, ovaries and tubes were found to be normal. Appendicectomy and omental biopsy was performed. Histology revealed a mesenteric fibromatosis-desmoid tumour.
\end{abstract}

Keywords Desmoid tumour · Fibromatosis · Appendix

\section{Introduction}

Desmoid tumours are rare non-encapsulated, locally invasive soft tissue tumours of fibrous origin that lack the ability to metastasise but are very notorious for recurrence; they are also referred to as fibromatoses. Desmoids are characterised histologically by spindle-shaped cells, surrounded by and separated from one another by abundant collagen [1]. These tumours arise in a variety of locations. Extra-abdominal desmoids occur in the chest wall, back, hip-gluteal region, shoulder girdle, upper arm and thigh. Intra-abdominal desmoids are rare tumours that can occur

V. Revicky $\cdot$ M. Freij $\cdot$ J. Nieto $\cdot$ E. P. Morris

Department of Obstetrics and Gynaecology,

Norfolk and Norwich University Hospital,

Colney Lane,

Norwich NR4 7UY, UK

V. Revicky $(\square)$

52 Atkinson Close,

Norwich NR5 9NE, UK

e-mail: revicky@yahoo.com in the pelvis and mesentery [2]. A mesenteric desmoid is a rare tumour that can occur in the bowel mesentery with an incidence of two to five per million [3]. The risk factor most strongly associated with desmoid tumours is familial adenomatous polyposis (FAP) $[4,5]$. This strong association is typically found with Gardner's syndrome [4-6]. Wellrecognised risk factors are trauma, female sex, oestrogens, pregnancy, trisomy 20 and 8 and the position of the APC germline mutation [2, 4-9]. Desmoids represent a major cause of morbidity and mortality in FAP patients [10]. Complications of desmoid tumours include bowel and ureteric obstruction, colectomy, ileorectal anastomosis and death.

Surgery is the main treatment, mainly for spontaneously arising desmoids [11, 12]. Medical therapy, chemotherapy, radiotherapy and anti-estrogen therapy may be used in case of inoperable tumours [6,13-18]. A possible future therapy is gene therapy by reintroduction of the APC gene into human fibroblasts [19]. Our report describes a case of a very rare desmoid tumour which rose from the mesentery of the appendix in a 17-year-old woman treated with surgery and who remained asymptomatic 12 months after the therapy.

\section{Case report}

A 17-year-old girl presented to an emergency admission unit with severe lower abdominal pain, lasting for 4 days prior to admission. The pain was episodic and radiated to the back. She described the intensity of pain as 8 out of 10 , requiring morphine analgesia. She had no history of nausea, vomiting or diarrhoea and no urinary symptoms. On physical examination, the abdomen was tender suprapubically and in the right iliac fossa, with no guarding or 
rebound. Her past medical and surgical history was insignificant, and she had no family history of familial adenomatous polyposis.

Pregnancy test was negative and urine analysis was within normal levels. An ultrasound scan demonstrated a large suprapubic mass measuring $7.6 \times 11 \times 8 \mathrm{~cm}$.

This was separate from the bladder and uterus which appeared normal and lay close to the right iliac vessels. The overall impression was that this was a large suprapubic mass with the appearance highly suspicious for a malignant mass likely to be of gynaecological origin, possibly of the right ovary. Magnetic resonance imaging (MRI) was performed. It confirmed the presence of the mass; the origin was difficult to identify with possibilities of a pedunculated ovarian tumour, possibly torsion or an unusual primary mesenteric or peritoneal tumour, possibly a sarcoma (Fig. 1). No lymphadenopathy within the pelvis or retroperitoneum was demonstrated. Tumour markers CA125, AFP, HCG and CEA were reported to be within normal limits.

This patient's case was discussed in the multidisciplinary gynaecological oncology meeting. She underwent an explorative laparotomy, which revealed a large mass arising from the appendix adherent to the omentum and to the posterior uterine wall, whereas the uterus, ovaries and tubes were found to be normal. Appendicectomy and omental biopsy were performed. The procedure and the postoperative course were uneventful.

Histology revealed that adjacent to the appendix was a non-encapsulated, relatively well-circumscribed spindle cell desmoids tumour with no cellular atypia, mitotic figures or

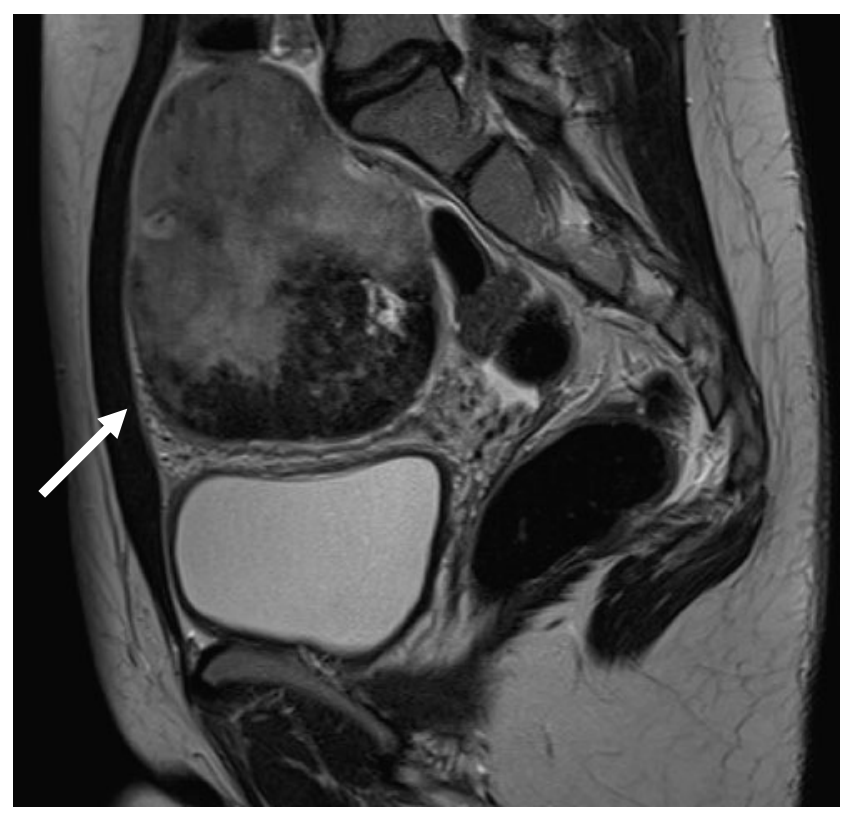

Fig. 1 MRI - there is an 8-cm heterogeneous solid mass arising in the pelvis necrosis and clean tissue margins of the specimen. At 12 months after surgery, the patient remains asymptomatic.

\section{Discussion}

Mesenteric desmoid tumours are rare, with an estimated annual incidence of two to five per million [3]. The tumours can arise sporadically or in association with familial adenomatous polyposis. Despite their inability to metastasise, desmoids are frequently locally invasive and may compress surrounding structures [6]. The clinical spectrum of desmoid disease ranges from incidental small, stable lesions to rapidly growing, huge abdominal masses. Patients may note the mass themselves or present with pain. Large desmoids can compress surrounding structures and cause an obstructive renal failure, a change in bowel habits or bowel obstruction. Rapidly growing tumours may cause weight loss, cachexia and malaise $[1,4,6]$. In our case, the woman did not have a family history of FAP or Gardner's syndrome and no history of trauma or a previous surgery. She did not notice the abdominal mass herself and did not have other common symptoms related to large desmoid tumours. She only presented with abdominal pain. Her pain was not caused by torsion of desmoid tumour as described in one previous case report of desmoid tumour rising from the mesentery of the appendix [12]. Estrogens have been implicated in the pathogenesis of sporadic desmoid tumours because females have a higher incidence, particularly those of childbearing age $[2,3]$. The growth of tumours in this group has been noted to be significantly faster than in males or in post- or pre-menopausal women, and one of these studies directly correlated the rate of growth of tumours to the level of endogenous estrogens [3]. In our case, woman's childbearing age may have been the only risk factor for developing a desmoid tumour. The optimal treatment has not yet been established and, in many cases, a multidisciplinary approach including surgery, chemotherapy and radiation therapy has been employed [6]. Based on a clinical course, desmoids can be divided into four groups. Church [20] described that $10 \%$ of tumours resolve spontaneously, 30\% undergo cycles of progression and resolution, $50 \%$ remain stable after diagnosis and $10 \%$ progress rapidly. This natural behaviour should be borne in mind when assessing the efficacy of therapy. Some desmoid tumours showed complete or partial regression and this may have happened anyway without any treatment $[20,21]$. Given the problems with the unpredictable growth rate of desmoids, there is a good case for observation of desmoids, particularly with no symptoms $[6,21]$. It is prudent to image all intra-abdominal tumours to ensure they do not compress ureters [6]. Symptomatic or rapidly growing desmoids usually require treatment [6]. Surgery 
is the main treatment, mainly for spontaneously arising desmoids $[11,12,22,23]$. The objective is to obtain tumour-free margins. A positive margin is a leading cause of recurrence [24].

Clark et al. [23] reported $36 \%$ mortality rate and $71 \%$ recurrence rate for patients operated on for mesenteric desmoids. Therefore, high rates of recurrence should be expected and patients must be counselled pre-operatively about this risk. Tzakis et al. [22] reported a technique where the tumour and small bowel were removed en bloc, perfused and cooled, and the tumour was resected in a bloodless field with subsequent auto-transplantation of the small bowel back into the patient. Medical therapy including NSAID, sulindac, chemotherapy including doxorubicin, radiotherapy and antiestrogen therapy with tamoxifen may be used in case of inoperable tumours [6, 13-18]. A possible future therapy is gene therapy by reintroduction of the APC gene into human fibroblasts [19]. Our patient was only 17 years old and presented with severe abdominal pain lasting for 4 days. She had no history and no symptoms related to a possible diagnosis of desmoid tumour. MRI did not help to distinguish the nature of the tumour either. The site of the tumour and the proximity of this structure to female internal genital organs caused difficulties to establishing a diagnosis. MRI concluded the tumour represented an unusual pelvic mass, with possibilities of a pedunculated ovarian tumour, possibly torsion or an unusual primary peritoneal tumour, possibly a sarcoma. However, a definitive diagnosis was established after surgery and histopathological examination of the tumour.

Given the unpredictable behaviour of desmoid tumours, it is wise to establish a follow-up plan [21]. Our patient had no family history of FAP or Gardner's syndrome, but it is prudent to consider colonoscopy or APC mutation testing in similar cases [25].

MRI scanning using T2-weighted imaging may be helpful in the monitoring of the behaviour of these tumours [26].

\section{Conclusion}

Desmoid tumours arise from various abdominal and extraabdominal locations.

Intra-abdominally, the most common site is within mesentery. In rare cases, the site of the desmoid tumour can be the mesentery of the appendix. The location of the tumour can cause diagnostic difficulties in identifying the source of pain. Surgery with complete tumour removal is commonly used in managing desmoid tumours. However, current management plans are not based on robust evidences. The rarity of this condition and the unpredictable behaviour of these tumours may complicate a design of randomised trials.

\section{References}

1. Middleton SB, Pack K, Phillips RK (2000) Telomere length in familial adenomatous polyposis-associated desmoids. Dis Colon Rectum 43:1535-1539

2. Reitamo JJ, Hayry P, Nykyri E et al (1982) The desmoid tumor. I. Incidence, sex-, age- and anatomical distribution in the Finnish population. Am J Clin Pathol 77:665-673

3. Reitamo JJ, Scheinin TM, Hayry P (1986) The desmoid syndrome. New aspects in the cause, pathogenesis and treatment of the desmoid tumor. Am J Surg 151:230-237

4. Gurbuz AK, Giardello FM, Petersen GM et al (1994) Desmoid tumors in familial adenomatous polyposis. Gut 35:377-381

5. Lofti AM, Dozois RR, Gordon H et al (1989) Mesenteric fibromatosis complicating familial adenomatous polyposis: predisposing factors and results of treatment. Int $\mathbf{J}$ Colorectal Dis 4:30-36

6. Julian N, Sturt H, Clark SK (2006) Current ideas in desmoid tumors. Familial Cancer 5:275-285

7. Hayry P, Reitamo JJ, Totterman S et al (1982) The desmoid tumor. II. Analysis of factors possibly contributing to the ethiology and growth behaviour. Am J Clin Pathol 77:674-680

8. Clark SK, Phillips RKS (1996) Desmoids in familial adenomatous polyposis. Br J Surg 83:1494-504

9. Caspari R, Olschwang S, Friedl W et al (1995) Familial adenomatous polyposis: desmoid tumors and lack of opthalmic lesions (CHRPE) associated with APC mutations beyond codon 1444. Hum Mol Genet 4:337-340

10. Nugent KP, Spigelman AD, Phillips RKS (1993) Life expectancy after colectomy and ileorectal anastomosis for familial adenomatous polyposis. Dis Colon Rectum 36:1059-1062

11. Middleton SB, Phillips RK (2000) Surgery for large intraabdominal tumors: report of four cases. Dis Colon Rectum 43:1759-1762

12. Furie DM, Patel U, Khan A et al (1991) Mesenteric desmoid of the appendix: a case report. Comput Med Imaging Graph 15:117120

13. Phillips RKS, Wallace MH, Lynch PM et al (2002) A randomised, double blind, placebo controlled study of celecoxib, a selective cyclooxygenase 2 inhibitor, on duodenal polyposis in familial adenomatous polyposis. Gut 50:857-860

14. Tsukada K, Church JM, Jagelman DG et al (1992) Noncytotoxic drug therapy for intra-abdominal desmoid tumor in patients with familial adenomatous polyposis. Dis Colon Rectum 35:2933

15. Patel SR, Evans HL, Benjamin RS (1993) Combination chemotherapy in adult desmoid tumors. Cancer 72:3244-3247

16. Gelmann EP (1997) Tamoxifen for the treatment of malignancies other than breast endometrial carcinoma. Semin Oncol 24:S1-6570

17. Janinis J, Patriki M, Vini L et al (2003) The pharmacological treatment of aggressive fibromatosis: a systematic review. Ann Oncol 14:181-190

18. Seiter K, Kemeny N (1993) Successful treatment of a desmoid tumor with doxorubicin. Cancer 71:2242-2244

19. Bright-Thomas RM, Agrawal A, Hargest R (2002) Preclinical studies of gene transfer for the treatment of desmoid disease in familial adenomatous polyposis. Br J Surg 89:1563-1569

20. Church JM (1995) Desmoid tumors in patients with familial adenomatous polyposis. Semin Colon Rectal Surg 6:29-32 
21. Jenkins NH, Freedman LS, McKibbin B (1986) Spontaneous regression of a desmoid tumor. J Bone Joint Surg Br 68:780781

22. Tzakis AG, Tryphonopoulos P, De Faria W et al (2003) Partial abdominal evisceration, ex vivo resection, and intestinal autotransplantation for the treatment of pathologic lesions of the root of the mesentery. J Am Coll Surg 197:770-776

23. Clark SK, Neale KF, Landgrebe JC et al (1999) Desmoid tumors complicating familial adenomatous polyposis. Br J Surg 86:11851189
24. Neeraj S, Rohit S, Sanford AD, Dy VC (2006) An unusual presentation of desmoid tumor in the ileum. The American Surgeon; 72, 9; ProQuest Medical Library, p 821, Sep

25. Sturt NJH, Gallagher MC, Bassett P et al (2004) Evidence for genetic predisposition to desmoid tumors in familial adenomatous polyposis (FAP) independent of the APC germline mutation. Gut 53:1832-1836

26. Healy JC, Reznek RH, Clark SK et al (1997) MRI appearances of desmoid tumors in familial adenomatous polyposis. AJR Am J Roentgenol 169:465-472 